\title{
Ueber diejenigen ebenen Curven, deren Coordinaten rationale Functionen eines Parameters sind.
}

\author{
(Von Herrn A. Clebsch zu Giessen.)
}

Die Classe von Abelschen Functionen, mit welcher eine algebraische ebene Curve $n^{\text {ter }}$ Ordnung zusammenhängt, wird durch die Zahl $p=\frac{n-1 . n-2}{2}$ bestimmt, wenn die Curve keine Doppel- und Rückkehrpunkte besitzt, und ich habe im $63^{\text {sten }}$ Bande dieses Journal pag. 189 eine Reihe von Resultaten angeführt, welche sich auf diese Bemerkung stützen. Dabei wurde vorzugsweise der Satz benutzt, dass die Summen gewisser Abelscher Integrale immer verschwinden, sobald eine Reihe von Puncten der gegebenen Curve den vollständigen Durchschnitt derselben mit einer andern algebraischen Curve bildet.

Wenn nun die Curve Doppelpunkte oder Rückkehrpunkte besitzt, so erniedrigt sich der Werth von $p$ um die Anzahl derselben; die erwähnten Integrale gehen zum Theil in Abelsche Integrale niederer Classe über, zum Theil aber auch in Integrale dritter Gattung. Die für die vorliegende Untersuchungen wichtigste Folgerung aus diesem Umstande ist, dass zwischen den Periodicitätsmoduln der modificirten Integrale lineare Relationen eintreten; und es ergeben sich daraus Erniedrigungen für die Zahlen, die ich a. a. 0. für die möglichen Lösungen der behandelten Probleme gefunden habe.

Statt die algebraischen Curven nach Ordnungen einzutheilen, und in diesen Unterabtheilungen zu machen nach der Anzahl der Doppel- und Rückkehrpunkte, welche dieselben aufweisen, kann man dieselben in Geschlechter eintheilen nach der Zahl $p$; zu dem ersten Geschlecht also alle diejenigen für welche $p=0$, zum zweiten diejenigen, für welche $p=1, u$. s. w. Dann erscheinen umgekehrt die verschiedenen Ordnungen als Unterabtheilungen in den Geschlechtern; und zwar kommt jede Ordnung in allen Geschlechtern vor bis zu $p=\frac{n-1 . n-2}{2}$, wo dann die allgemeinste, d. h. von Doppel- und Rückkehrpunkten völlig freie Curve $n^{\text {ter }}$ Ordnung ihre Stelle findet.

Die zu demselben Geschlechte gehörigen Curven kann man, wie a. a. 0. gezeigt ist, auch dadurch definiren, dass ihre homogenen Coordinaten sich als rationale ganze Functionen von zwei Parametern $s, z$ darstellen lassen, zwischen 
44 Clebsch, über Curven, deren Coord. rationale Funct. eines Parameters sind.

denen eine algebraische Gleichung

$$
f(s, z)=0
$$

besteht, die eine entsprechende Classe Abelscher Functionen begründet. Insbesondere für $p=0$ kann man $s$ durch $z$ rational ausdrücken; für $p=1$ erfordert diese Darstellung die Quadratwurzel aus einem Ausdruck dritten oder vierten Grades, u. s. w.

In diesem Aufsatze werde ich mich mit dem ersten Geschlechte beschäftigen, für welches $p=0$, für welches also die Doppel- und Rückkehrpunkte die ihrer Ordnung nach höchste erreichbare Anzáhl besitzen. Diese Curven hat Herr Salmon bereits in seinem "Treatise on higher plane curves" kurz discutirt (p. 94). Ich werde einige weitere Eigenschaften derselben angeben.

Dass jede Curve $n^{\text {ter }}$ Ordnung, welche $\frac{n-1 . n-2}{2}$ Doppelpunkte besitzt, wirklich die rationale Darstellung ihrer Coordinaten durch einen Parameter gestattet, sieht man auf folgende Weise ein. Durch die Doppelpunkte und durch $\frac{h . h+3}{2}-\frac{n-1 . n-2}{2}$ andere Punkte der Curve ist, wenn $h<n$, eine Curve $h^{\text {ter }}$ Ordnung vollständig bestimmt. Damit diese Punkte zugleich den vollständigen Durchschnitt beider Curven bilden, muss

$$
\frac{h \cdot h+3}{2}+\frac{n-1 \cdot n-2}{2}=h n
$$

sein, d. h. $h=n-1$ oder $h=n-2$.

Legt man also ein System $u+\lambda v=0$ von Curven $(n-1)^{\text {ter }}$ Ordnung durch die Doppelpunkte und durch' $2 n-3$ feste Punkte der gegebenen Curve, so schneidet jede Curve des Systems die gegebene noch in einem beweglichen Punkte. Man muss also die Coordinaten dieses Punktes durch $\lambda$ rational ausdrücken können.

Legt man ebenso ein System $u+\lambda v=0$ von Curven $(n-2)^{\text {ter }}$ Ordnung durch die Doppelpunkte und durch $n-3$ feste Punkte der gegebenen Curve, so schneidet abermals jede Curve des Systems die gegebene Curve nur noch in einem beweglichen Punkte, und man kann also die Coordinaten dieses Punktes durch $\lambda$ rational ausdrücken.

So kann man also diese Darstellung auf zwei verschiedene Arten in jedem Falle sofort leisten. 


\section{\$. 1.}

Die Singularitäten derjenigen Curven, deren Coordinaten rationale Functionen eines Parameters sind.

Betrachten wir eine Curve, deren homogene Coordinaten in der Form ausdrückbar sind:

$$
\text { (1.) } \quad\left\{\begin{array}{l}
x_{1}=f_{1}(\lambda, \mu), \\
x_{2}=f_{2}(\lambda, \mu), \\
x_{3}=f_{3}(\lambda, \mu),
\end{array}\right.
$$

wo $f_{1}, f_{2}, f_{3}$ ganze homogene Functionen $n^{\text {ter }}$ Ordnung von $\lambda$ und $\mu$ sind, welche sich nicht als homogene Functionen niedrigerer Ordnung von zwei ganzen rationalen Functionen von $\lambda, \mu$ darstellen lassen. Jedem Punkte der Curve entspricht dann ein gewisser Werth des Verhältnisses $\frac{\lambda}{\mu}$, und im Allgemeinen auch nur ein einziger. Die Functionen $f_{1}, f_{2}, f_{3}$ dürfen als von einem allen gemeinschaftlichen Factor frei angesehen werden. Die Curve ist daher von der $n^{\text {ten }}$ Ordnung, da jede Linie

$$
u_{1} x_{1}+u_{2} x_{2}+u_{3} x_{3}=0
$$

sie in den $n$ Punkten schneidet, deren Parameter sich aus der Gleichung $n^{\text {ten }}$ Grades:

$$
\text { (2.) } u_{1} f_{1}+u_{2} f_{2}+u_{3} f_{3}=0
$$

ergeben.

Die Gleichung der Curve in Liniencoordinaten findet man, wenn man die Discriminante von (2.) bildet, also wenn man $\lambda$ und $\mu$ aus den beiden Gleichungen

$$
\left\{\begin{array}{l}
u_{1} \frac{\partial f_{1}}{\partial \lambda}+u_{2} \frac{\partial f_{2}}{\partial \lambda}+u_{3} \frac{\partial f_{3}}{\partial \lambda}=0 \\
u_{1} \frac{\partial f_{1}}{\partial \mu}+u_{2} \frac{\partial f_{2}}{\partial \mu}+u_{3} \frac{\partial f_{3}}{\partial \mu}=0
\end{array}\right.
$$

eliminirt. Diese Discriminante ist im Allgemeinen von dem Grade $2(n-1)$, und also die Curve im Allgemeinen von der $2(n-1)^{\text {ten }}$ Classe. Aber insbesondere können solche Werthe von $\lambda, \mu$ existiren, für welche

$$
\text { (4.) }\left\{\begin{array}{l}
l \frac{\partial f_{1}}{\partial \lambda}=m \frac{\partial f_{1}}{\partial \mu}=a_{1}, \\
l \frac{\partial f_{2}}{\partial \lambda}=m \frac{\partial f_{2}}{\partial \mu}=a_{2}, \\
l \frac{\partial f_{3}}{\partial \dot{\lambda}}=m \frac{\partial f_{3}}{\partial \mu}=a_{3} .
\end{array}\right.
$$


Die Discriminante muss dann nothwendig den Factor $u_{1} a_{1}+u_{2} a_{2}+u_{3} a_{3}$ enthalten, durch dessen Verschwinden beide Gleichungen $(2$.) befriedigt werden. Die $a$ sind nach (1.) die zu einem solchen Parameter $\frac{\lambda}{\mu}$ gehörigen Coordinaten. Ist $\varkappa$ die Anzahl von Lösungen, welche die Gleichungen (4.) zulassen, so ist die Classe der Curve $2(n-1)-\varkappa$.

Aus den Gleichungen (3.) ergeben sich für die Coordinaten der Tangente (oder für die den Gleichungen (1.) analoge Darstellung der Curve in Liniencoordinaten):

$$
\left\{\begin{array}{l}
\boldsymbol{\rho} u_{1}=\frac{\partial f_{2}}{\partial \lambda} \frac{\partial f_{3}}{\partial \mu}-\frac{\partial f_{3}}{\partial \lambda} \frac{\partial f_{2}}{\partial \mu} \\
\rho u_{2}=\frac{\partial f_{3}}{\partial \lambda} \frac{\partial f_{1}}{\partial \mu}-\frac{\partial f_{1}}{\partial \lambda} \frac{\partial f_{3}}{\partial \mu} \\
\rho u_{3}=\frac{\partial f_{1}}{\partial \lambda} \frac{\partial f_{2}}{\partial \mu}-\frac{\partial f_{2}}{\partial \lambda} \frac{\partial f_{1}}{\partial \mu}
\end{array}\right.
$$

so dass die Gleichung der Tangente folgende ist:

$$
\text { (6.) }\left|\begin{array}{lll}
\boldsymbol{X}_{1} & \boldsymbol{X}_{2} & \boldsymbol{X}_{3} \\
\frac{\partial f_{1}}{\partial \lambda} & \frac{\partial f_{2}}{\partial \lambda} & \frac{\partial f_{3}}{\partial \lambda} \\
\frac{\partial f_{1}}{\partial \mu} & \frac{\partial f_{2}}{\partial \mu} & \frac{\partial f_{3}}{\partial \mu}
\end{array}\right|=\mathbf{0}
$$

Dieser Ausdruck wird unbestimmt, wenn $\lambda, \mu$ ein System von Lösungen der Gleichungen (4.) sind. Dividirt man aber in der Gleichung (6.), welche für den Augenblick durch $\Omega=0$ bezeichnet sein mag, zuerst durch einen der Coefficienten, so nehmen die anderen die Form $\frac{0}{0}$ an, und indem man dann nach der gewöhnlichen Regel entweder nach $\lambda$ oder nach $\mu$ differentiirt, mit dem reducirten Nenner aber wieder heraufmultiplicirt, findet man die Gleichung der Tangente in der Form $\frac{\partial \Omega}{\partial \lambda}=0$ oder $\frac{\partial \Omega}{\partial \mu}=0$, oder endlich, wenn $p, q$ beliebige Grössen sind, in der Form

$$
p \frac{\partial \boldsymbol{\Omega}}{\partial \lambda}+q \frac{\partial \boldsymbol{\Omega}}{\partial \boldsymbol{\mu}}=\mathbf{0} .
$$

Die Ausführung für die Function $\Omega$ giebt dann:

$$
0=\left|\begin{array}{ccc}
X_{1} & X_{2} & X_{3} \\
p \frac{\partial^{2} f_{1}}{\partial \lambda^{2}}+q \frac{\partial^{2} f_{1}}{\partial \lambda \partial \mu} & p \frac{\partial^{2} f_{2}}{\partial \lambda^{2}}+q \frac{\partial^{2} f_{2}}{\partial \lambda \partial \mu} & p \frac{\partial^{2} f_{3}}{\partial \lambda^{2}}+q \frac{\partial^{2} f_{3}}{\partial \lambda \partial \mu} \\
p \frac{\partial^{2} f_{1}}{\partial \lambda \partial \mu}+q \frac{\partial^{2} f_{1}}{\partial \mu^{2}} & p \frac{\partial^{2} f_{2}}{\partial \lambda \partial \mu}+q \frac{\partial^{2} f_{2}}{\partial \mu^{2}} & p \frac{\partial^{2} f_{3}}{\partial \lambda \partial \mu}+q \frac{\partial^{2} f_{3}}{\partial \mu^{2}}
\end{array}\right|
$$


oder, was dasselbe ist:

$$
\mathbf{0}=\left|\begin{array}{cccc}
\frac{\partial^{2} f_{1}}{\partial \lambda^{2}} & \frac{\partial^{2} f_{2}}{\partial \lambda^{2}} & \frac{\partial^{2} f_{3}}{\partial \lambda^{2}} & q^{2} \\
\frac{\partial^{2} f_{1}}{\partial \lambda \partial \mu} & \frac{\partial^{2} f_{2}}{\partial \lambda \partial \mu} & \frac{\partial^{2} f_{3}}{\partial \lambda \partial \mu} & -p q \\
\frac{\partial^{2} f_{1}}{\partial \mu^{2}} & \frac{\partial^{2} f_{2}}{\partial \mu^{2}} & \frac{\partial^{2} f_{3}}{\partial \mu^{2}} & p^{2} \\
X_{1} & X_{2} & X_{3} & 0
\end{array}\right| .
$$

Nun kann man die Gleichungen (4.) auch in die Gestalt bringen:

$$
\text { (7.) }\left\{\begin{array}{l}
l \lambda \frac{\partial^{2} f_{1}}{\partial \lambda^{2}}+(l \mu-m \lambda) \frac{\partial^{2} f_{1}}{\partial \lambda \partial \mu}-m \mu \frac{\partial^{2} f_{1}}{\partial \mu^{2}}=0, \\
l \lambda \frac{\partial^{2} f_{2}}{\partial \lambda^{2}}+(l \mu-m \lambda) \frac{\partial^{2} f_{2}}{\partial \lambda \partial \mu}-m \mu \frac{\partial^{2} f_{2}}{\partial \mu^{2}}=0 \\
l \lambda \frac{\partial^{2} f_{3}}{\partial \lambda^{2}}+(l \mu-m \lambda) \frac{\partial^{2} f_{3}}{\partial \lambda \partial \mu}-m \mu \frac{\partial^{2} f_{3}}{\partial \mu^{2}}=0 .
\end{array}\right.
$$

Aus diesen folgt, dass erstlich die Determinante

$$
\text { (8.) } \quad \Delta=\left|\begin{array}{lll}
\frac{\partial^{2} f_{1}}{\partial \lambda^{2}} & \frac{\partial^{2} f_{1}}{\partial \lambda \partial \mu} & \frac{\partial^{2} f_{1}}{\partial \mu^{2}} \\
\frac{\partial^{2} f_{2}}{\partial \lambda^{2}} & \frac{\partial^{2} f_{2}}{\partial \lambda \partial \mu} & \frac{\partial^{2} f_{2}}{\partial \mu^{2}} \\
\frac{\partial^{2} f_{3}}{\partial \lambda^{2}} & \frac{\partial^{2} f_{3}}{\partial \lambda \partial \mu} & \frac{\partial^{2} f_{3}}{\partial \mu^{2}}
\end{array}\right|
$$

für die betreffenden Werthe $\frac{\lambda}{\mu}$ immer verschwindet; und zweitens, dass sich immer drei Grössen $r_{1}, r_{2}, r_{3}$ so bestimmen lassen, dass

$$
\text { (9.) }\left\{\begin{array}{l}
\frac{\partial \Delta}{\partial \frac{\partial^{2} f_{i}}{\partial \lambda^{2}}}=l \lambda . r_{i}, \\
\frac{\partial \Delta}{\partial \frac{\partial^{2} f_{i}}{\partial \lambda \partial \mu}}=(l \mu-m \lambda) r_{i} \\
\frac{\partial \Delta}{\partial \frac{\partial^{2} f_{i}}{\partial \mu^{2}}}=-m \mu . r_{i} .
\end{array}\right.
$$

Durch die Einführung dieser Werthe geht endlich, mit Uebergehung eines unwesentlichen Factors $(p \lambda-q \mu)(p l+q m)$ die Gleichung der Tangente für einen solchen Punkt in die bestimmte Form über:

$$
\text { (10.) } X_{1} r_{1}+X_{2} r_{2}+X_{3} r_{3}=0 \text {. }
$$


48 Clebsch, über Curven, deren Coord. rationale Funct. eines Parameters sind.

Doppelpunkte der Curve sind diejenigen, für welche zwei Werthsysteme $\lambda, \mu ; \lambda^{\prime}, \mu^{\prime}$ dieselben Werthe der Coordinaten ergeben. Man hat also für sie

$$
\left\{\begin{array}{l}
\varkappa f_{1}(\lambda, \mu)=\varkappa^{\prime} f_{1}\left(\lambda^{\prime}, \mu^{\prime}\right), \\
\varkappa f_{2}(\lambda, \mu)=\varkappa^{\prime} f_{2}\left(\lambda^{\prime}, \mu^{\prime}\right), \\
\varkappa f_{3}(\lambda, \mu)=\varkappa^{\prime} f_{3}\left(\lambda^{\prime}, \mu^{\prime}\right) .
\end{array}\right.
$$

Setzen wir nun

$$
\frac{f_{2}(\lambda ; \mu) \cdot f_{3}\left(\lambda^{\prime}, \mu^{\prime}\right)-f_{3}(\lambda, \mu) \cdot f_{2}\left(\lambda^{\prime}, \mu^{\prime}\right)}{\lambda \mu^{\prime}-\lambda^{\prime} \mu}=\Phi_{1}\left(\lambda, \mu ; \lambda^{\prime}, \mu^{\prime}\right),
$$

u. s. w.

wo denn $\Phi_{1}, \Phi_{2}, \Phi_{3}$ symmetrische Functionen der Werthepaare $\lambda, \mu ; \lambda^{\prime}, \mu^{\prime}$ sind, und zwar für jedes Werthepaar homogen von $\operatorname{der}(n-1)^{\text {ten }}$ Ordnung, so bestehen die identischen Gleichungen:

$$
\left\{\begin{array}{l}
\Phi_{1} \cdot f_{1}(\lambda, \mu)+\Phi_{2} \cdot f_{2}(\lambda, \mu)+\Phi_{3} \cdot f_{3}(\lambda, \mu)=0, \\
\Phi_{1} \cdot f_{1}\left(\lambda^{\prime}, \mu^{\prime}\right)+\Phi_{2} \cdot f_{2}\left(\lambda^{\prime}, \mu^{\prime}\right)+\Phi_{3} \cdot f_{3}\left(\lambda^{\prime}, \mu^{\prime}\right)=0 .
\end{array}\right.
$$

Wenn man aus $\Phi_{1}, \Phi_{2}$ etwa $\lambda^{\prime}, \mu^{\prime}$ eliminirt, so ergiebt sich für $\frac{\lambda}{\mu}$ eine Gleichung des Grades $2(n-1)^{2}$. Für die Lösungen derselben und die zugehörigen Werthe von $\frac{\lambda^{\prime}}{\mu^{\prime}}$ verschwindet nun entweder auch $\Phi_{3}$; und dann hat man einen Doppelpunkt vor sich; oder es verschwinden $f_{3}^{\prime}(\lambda, \mu), f_{3}\left(\lambda^{\prime}, \mu^{\prime}\right)$, und die betreffende Lösung ist der Frage fremd. In der That zieht das Verschwinden der letzten beiden Grössen, wie man aus (12.) sieht, das Verschwinden von $\Phi_{1}, \Phi_{2}$ nach sich, ausser wenn $\frac{\lambda}{\mu}=\frac{\lambda^{\prime}}{\mu^{\prime}}$. Es entstehen also so $n(n-1)$ Lösungen der Gleichungen $\Phi_{1}, \Phi_{2}$, welche der Frage fremd sind, und es bleiben also $n-1 . n-2$ Werthe von $\frac{\lambda}{\mu}$ übrig, welche Doppelpunkten entsprechen, oder es giebst $\frac{n-1 . n-2}{2}$ Doppelpunkte auf der Curve, wie dies a priori aus der Grösse der Zahl $p$ klar ist.

Die Elimination von $\frac{\lambda}{\mu}, \frac{\lambda^{\prime}}{\mu^{\prime}}$ aus $\Phi_{1}=0, \Phi_{2}=0$ muss nach dem Obigen zunächst auf eine Gleichung der Form

$$
\frac{\lambda^{\prime}}{\mu^{\prime}}=F\left(\frac{\lambda}{\mu}\right)
$$

führen; sodann aber auf die Endgleichung:

$$
\left(f_{3}(\lambda, \mu)\right)^{m-1} \cdot \psi(\lambda, \mu)=0,
$$


wo nun $\psi=0$ die gesuchte Gleichung $n-1 . n-2^{\text {ten }}$ Grades ist. Aber die Lösung derselben erfordert nur die Lösung einer Gleichung des $\frac{n-1 \cdot n-2^{\text {ten }}}{2}$ Grades. Denn ausser durch $\frac{\lambda}{\mu}$ wird diese Gleichung auch noch durch $\frac{\lambda^{\prime}}{\mu^{\prime}}=F\left(\frac{\lambda}{\mu}\right)$ erfüllt, wo $\frac{\lambda^{\prime}}{\mu^{\prime}}$ zu demselben Doppelpunkt gehört, welcher durch $\frac{\lambda}{\mu}$ bezeichnet wird. Es ist daher auch nothwendig

$$
\frac{\lambda}{\mu}=\boldsymbol{F}\left(\frac{\lambda^{\prime}}{\mu^{\prime}}\right) \text {, }
$$

und die Gleichung $\psi=0$ kann daher nach Abel auf eine Gleichung $\frac{n-1 . n-2^{\text {len }}}{2}$ Grades, und auf $\frac{n-1 . n-2}{2}$ quadratische Gleichungen zurückgeführt werden.

Ich will jetzt untersuchen, unter welchen Umständen ein Doppelpunkt in einen Rückkehrpunkt übergehen kann. Für diesen Fall muss die Gleichung (6.) der Tangente für beide im Doppelpunkt sich schneidende Zweige dieselbe sein, man muss also die Gleichungen haben:

$$
\begin{aligned}
& \left(\frac{\partial f_{2}}{\partial \lambda} \frac{\partial f_{3}}{\partial \mu}-\frac{\partial f_{3}}{\partial \lambda} \frac{\partial f_{2}}{\partial \mu}\right)=m\left(\frac{\partial f_{2}}{\partial \lambda^{\prime}} \frac{\partial f_{3}}{\partial \mu^{\prime}}-\frac{\partial f_{3}}{\partial \lambda^{\prime}} \frac{\partial f_{2}}{\partial \mu^{\prime}}\right), \\
& \left(\frac{\partial f_{3}}{\partial \lambda} \frac{\partial f_{1}}{\partial \mu}-\frac{\partial f_{1}}{\partial \lambda} \frac{\partial f_{3}}{\partial \mu}\right)=m\left(\frac{\partial f_{3}}{\partial \lambda^{\prime}} \frac{\partial f_{1}}{\partial \mu^{\prime}}-\frac{\partial f_{1}}{\partial \lambda^{\prime}} \frac{\partial f_{3}}{\partial \mu^{\prime}}\right), \\
& \left(\frac{\partial f_{1}}{\partial \lambda} \frac{\partial f_{2}}{\partial \mu}-\frac{\partial f_{2}}{\partial \lambda} \frac{\partial f_{1}}{\partial \mu}\right)=m\left(\frac{\partial f_{1}}{\partial \lambda^{\prime}} \frac{\partial f_{2}}{\partial \mu^{\prime}}-\frac{\partial f_{2}}{\partial \lambda^{\prime}} \frac{\partial f_{1}}{\partial \mu^{\prime}}\right),
\end{aligned}
$$

wo die Functionen $f$ links mit den Variablen $\lambda, \mu$, rechts mit den Variablen $\lambda^{\prime}, \iota^{\prime}$ zu nehmen sind. Aus diesen Gleichungen folgt:

$$
\left|\begin{array}{lll}
\frac{\partial f_{1}}{\partial \lambda^{\prime}} & \frac{\partial f_{1}}{\partial \lambda} & \frac{\partial f_{1}}{\partial \mu} \\
\frac{\partial f_{2}}{\partial \lambda^{\prime}} & \frac{\partial f_{2}}{\partial \lambda} & \frac{\partial f_{2}}{\partial \mu} \\
\frac{\partial f_{3}}{\partial \lambda^{\prime}} & \frac{\partial f_{3}}{\partial \lambda} & \frac{\partial f_{3}}{\partial \mu}
\end{array}\right|=\mathbf{0 ,} \quad\left|\begin{array}{lll}
\frac{\partial f_{1}}{\partial \mu^{\prime}} & \frac{\partial f_{1}}{\partial \lambda} & \frac{\partial f_{1}}{\partial \mu} \\
\frac{\partial f_{2}}{\partial \mu^{\prime}} & \frac{\partial f_{2}}{\partial \lambda} & \frac{\partial f_{2}}{\partial \mu} \\
\frac{\partial f_{3}}{\partial \mu^{\prime}} & \frac{\partial f_{3}}{\partial \lambda} & \frac{\partial f_{3}}{\partial \mu}
\end{array}\right|=\mathbf{0 .}
$$

Aber diese Gleichungen sagen nicht Anderes aus, als dass $\frac{\lambda}{\mu}$ und $\frac{\lambda^{\prime}}{\mu^{\prime}}$ Doppelwurzeln der Gleichung seien, von welcher die Parameter der Doppelpunkte abhängen. Dies sieht man sofort, wenn man die Bedingung aufstellt, dass die Gleichungen (11.) ausser für $\varkappa, \varkappa^{\prime}, \lambda, \mu, \lambda^{\prime}, \mu^{\prime}$ auch noch für $x+d x, x^{\prime}$, $\lambda+d \lambda, \mu, \lambda^{\prime}+d \lambda^{\prime}, \mu^{\prime}$ und für $\varkappa+d \varkappa, \varkappa^{\prime}, \lambda, \mu+d \mu, \lambda^{\prime}, \mu^{\prime}+d \mu^{\prime}$ bestehen sollen. Hiebei sind also zwei verschiedene Fälle zu unterscheiden. Einmal nämlich 
50 Clebsch, über Curven, deren Coord. rationale Funct. eines Parameters sind.

können $\frac{\lambda}{\mu}, \frac{\lambda^{\prime}}{\mu^{\prime}}$ zwei verschiedene Doppelwurzeln sein, und zweitens können $\frac{\lambda}{\mu}, \frac{\lambda^{\prime}}{\mu^{\prime}}$ einander gleich werden und so das Paar gleicher Wurzeln repräsentiren. Im ersten Falle tritt kein Rückkehrpunkt ein, sondern zwei unendlich nahe Doppelpunkte, wobei denn freilich auch die beiden Tangenten in eine zusammenfallen. Dieses Vorkommen erfordert zwei Bedingungen zwischen den Coefficienten, und führt keine weitere Reduction der Classe mit sich, als diesen zwei Doppelpunkten überhaupt zukommt. Im zweiten Falle dagegen erhält man einen wirklichen Rückkehrpunkt, und zugleich eine Reduction der Classe. Ist nämlich $\frac{\lambda^{\prime}}{\mu^{\prime}}=\frac{\lambda}{\mu}+\varepsilon$, und convergirt $\varepsilon$ gegen Null, so gehen die Gleichungen (14.) in die eine Gleichung (8.)

$$
\Delta=0
$$

über. Es wird also wirklich nur eine Bedingung erfordert. Zugleich gehen die Gleichungen (11.) in die Gleichungen (4.) über. Die oben betrachteten ^ Punkte, für welche eine Reduction der Classe eintritt, fallen also in der That mit den Rückkehrpunkten zusammen.

Nicht blos $\Delta$ selbst verschwindet für jeden Rückkehrpunkt, sondern auch sein Differential, so dass der einem Rückkehrpunkte entsprechende Parameter jederzeit eine Doppelwurzel von $\boldsymbol{A}=0$ ist. Man hat nämlich:

$$
\begin{gathered}
d \Delta=\frac{\partial \Delta}{\partial \lambda} d \lambda+\frac{\partial \Delta}{\partial \mu} d \mu \\
=\Sigma_{i}\left\{\frac{\partial \Delta}{\partial \frac{\partial^{2} f_{i}}{\partial \lambda^{2}}} d \frac{\partial^{2} f_{i}}{\partial \lambda^{2}}+\frac{\partial \Delta}{\partial \frac{\partial^{2} f_{i}}{\partial \lambda \partial \mu}} d \frac{\partial^{2} f_{i}}{\partial \lambda \partial \mu}+\frac{\partial \Delta}{\partial \frac{\partial^{2} f_{i}}{\partial \mu^{2}}} d \frac{\partial^{2} f_{i}}{\partial \mu^{2}}\right\}
\end{gathered}
$$

oder nach (9.):$$
=\Sigma_{i} r_{i}\left\{l \lambda d \frac{\partial^{2} f_{i}}{\partial \lambda^{2}}+(l \mu-m \lambda) d \frac{\partial^{2} f_{i}}{\partial \lambda \partial \mu}-m \mu d \frac{\partial^{2} f_{i}}{\partial \mu^{2}}\right\}
$$$$
=(n-2) \Sigma_{i} r_{i}\left(l d \frac{\partial f_{i}}{\partial \lambda}-m d \frac{\partial f_{i}}{\partial \mu}\right) \text {. }
$$

Und dieser Ausdruck verschwindet, da nach (8.), (9.) folgende Gleichungen bestehen :

$$
\begin{aligned}
& \Sigma r_{i} \frac{\partial^{2} f_{i}}{\partial \lambda^{2}}=0 \\
& \Sigma r_{i} \frac{\partial^{2} f_{i}}{\partial \lambda \partial \mu}=0 \\
& \Sigma r_{i} \frac{\partial^{2} f_{i}}{\partial \mu^{2}}=0 .
\end{aligned}
$$


Diese Betrachtungen werden von Wichtigkeit bei der Bestimmung der Wendepunkte. Sind $\frac{\lambda}{\mu}, \frac{\lambda^{\prime}}{\mu^{\prime}}, \frac{\lambda^{\prime \prime}}{\mu^{\prime \prime}}$ die Parameter dreier Punkte der Curve, welche auf einer Geraden liegen, so müssen dieselben der Bedingung genügen:

$$
\left\{\begin{aligned}
0 & =\frac{\left|\begin{array}{lll}
f_{1}(\lambda, \mu) & f_{1}\left(\lambda^{\prime}, \mu^{\prime}\right) & f_{1}\left(\lambda^{\prime \prime}, \mu^{\prime \prime}\right) \\
f_{2}(\lambda, \mu) & f_{2}\left(\lambda^{\prime}, \mu^{\prime}\right) & f_{2}\left(\lambda^{\prime \prime}, \mu^{\prime \prime}\right) \\
f_{3}(\lambda, \mu) & f_{3}\left(\lambda^{\prime}, \mu^{\prime}\right) & f_{3}\left(\lambda^{\prime \prime}, \mu^{\prime \prime}\right)
\end{array}\right|}{\lambda^{\prime} \mu^{\prime \prime}-\mu^{\prime} \lambda^{\prime \prime} \cdot \lambda^{\prime \prime} \mu-\mu^{\prime \prime} \lambda . \lambda \mu^{\prime}-\mu \lambda^{\prime}} \\
= & \Psi\left(\lambda, \mu ; \lambda^{\prime}, \mu^{\prime} ; \lambda^{\prime \prime}, \mu^{\prime \prime}\right) .
\end{aligned}\right.
$$

Für einen Wendepunkt muss diese Gleichung erfüllt sein, wenn man $\frac{\lambda}{\mu}, \frac{\lambda^{\prime}}{\mu^{\prime}}$ $\frac{\lambda^{\prime \prime}}{\mu^{\prime \prime}}$ unendlich wenig von einander verschieden annimmt, und da zu gleicher Zeit die Gleichung (15.) in

$$
-\frac{A}{2 n(n-1)^{2}}=0
$$

übergeht, so findet man die Wendepunkte aus der Gleichung $\Delta=0$. Die Zahl der Wendepunkte beträgt somit im Allgemeinen $3(n-2)$. Aber da, wie oben bewiesen, der Parameter jedes Rückkehrpunkts eine Doppelwurzel dieser Gleichung wird, so ist, wenn « Doppelpunkte zu Rückkehrpunkten werden, die Anzahl der übrigbleibenden Wendepunkte $3(n-2)-2 \%$.

Diese Zahl giebt eine obere Grenze für die Anzahl von Rückkehrpunkten, welche eine Curve besitzen kann. Man sieht nämlich daraus, dass die Anzahl der Rückkehrpunkte einer Curve $n^{\text {ter }}$ Ordnung die Zahl $\frac{3}{2}(n-2)$ niemals übersteigen kann. Es scheint, dass diese Grenze (oder vielmehr die grösste darin enthaltene ganze Zahl) wirklich erreicht werden kann; wenigstens tritt dies bei den Curven dritter und vierter Ordnung ein.

Die Berührungspunkte der Tangenten, welche sich von einem äusseren Punkte an die Curve ziehen lassen, findet man aus (6.) mit Hülfe einer Gleichung $2 n-2^{\text {ten }}$ Grades für $\frac{\lambda}{\mu}$. Ist aber in (5.) $X$ ein Punkt der Curve selbst, und bezeichnen wir seine Parameter durch $\lambda^{0}, \mu^{0}$, ebenso die Functionen $f$ für diese Werthe durch denselben Index, so geht die Gleichung (6.) über in:

$$
\text { (16.) }\left|\begin{array}{lll}
f_{1}^{\prime \prime} & \frac{\partial f_{1}}{\partial \lambda} & \frac{\partial f_{1}}{\partial \mu} \\
f_{2}^{(0} & \frac{\partial f_{2}}{\partial \lambda} & \frac{\partial f_{2}}{\partial \mu} \\
f_{3}^{\prime} & \frac{\partial f_{3}}{\partial \lambda} & \frac{\partial f_{3}}{\partial \mu}
\end{array}\right|=\mathbf{0}
$$


was durch $\left(\lambda \mu^{0}-\lambda^{0} \mu\right)^{2}$ theilbar ist, und mit der Gleichung

$$
0=\left(\lambda, \mu^{0}-\lambda^{0} \mu\right)^{2} . \Psi\left(\lambda, \mu ; \lambda, \mu ; \lambda^{0}, \mu^{0}\right)
$$

identisch wird. Nach Entfernung des ausgeschiedenen Factors bleibt eine Gleichung

$$
\text { (17.) } \Psi\left(\lambda, \mu ; \lambda, \mu ; \lambda^{0}, \mu^{0}\right)=0
$$

vom $2 n-4^{\text {ten }}$ Grade übrig; sie giebt die Berührungspunkte der $2 n-4$ Tangenten, welche man von einem Punkte der Curve noch ziehen kann. Aber die Gleichung (16.) ist durch die Werthe von $\lambda, \mu$ erfüllt, welche den Gleichungen (4.) genügen. Die Anzahl der wirklichen Tangenten bleibt also $2 n-4-x$.

Ist insbesondere der Punkt der Curve ein Wendepunkt, so ist

$$
\Psi\left(\lambda^{0}, \mu^{0} ; \lambda^{0}, \mu^{0} ; \lambda^{0}, \mu^{0}\right)=0 .
$$

Daher enthält die Gleichung (17.) noch eine weitere Wurzel $\frac{\lambda^{0}}{\mu^{0}}$, und die Anzahl der wirklichen Tangenten bleibt $2 n-5-x$.

Ist dagegen der Punkt der Curve ein Doppelpunkt, und ist $\frac{\lambda^{(1)}}{\mu^{(1)}}$ der andere dem Doppelpunkte entsprechende Werth des Parameters, so kann man in (16.) statt $f^{(0)}$ auch $f^{(1)}$ schreiben, und $\frac{\lambda}{\mu}=\frac{\lambda^{(1)}}{\mu^{(1)}}$ ist daher auch eine Doppelwurzel von (16.). Von einem Doppelpunkt lassen sich also $2 n-6-\varkappa$ Tangenten an die Curve ziehen.

Ist endlich der Punkt der Curve ein Rückkehrpunkt, von welchem aus Tangenten gezogen werden sollen, so ist $\frac{\lambda^{0}}{\mu^{0}}$ eine vierfache Wurzel der Gleichung (16.). In der That, bildet man etwa den dritten Differentialquotienten des Ausdrucks der linken Seite von (16.) nach $\lambda$, so erhält man:

$$
\begin{gathered}
\left|\begin{array}{lll}
f_{1}^{0} & \frac{\partial^{4} f_{1}}{\partial \lambda^{4}} & \frac{\partial f_{1}}{\partial \mu} \\
f_{2}^{0} & \frac{\partial^{4} f_{2}}{\partial \lambda^{4}} & \frac{\partial f_{2}}{\partial \mu} \\
f_{3}^{0} & \frac{\partial^{4} f_{3}}{\partial \lambda^{4}} & \frac{\partial f_{3}}{\partial \mu}
\end{array}\right|+3\left|\begin{array}{lll}
f_{1}^{0} & \frac{\partial^{3} f_{1}}{\partial \lambda^{3}} & \frac{\partial^{2} f_{1}}{\partial \lambda \partial \mu} \\
f_{2}^{0} & \frac{\partial^{3} f_{2}}{\partial \lambda^{3}} & \frac{\partial^{2} f_{2}}{\partial \lambda \partial \mu} \\
f_{3}^{0} & \frac{\partial^{3} f_{3}}{\partial \lambda^{3}} & \frac{\partial^{2} f_{3}}{\partial \lambda \partial \mu}
\end{array}\right| \\
+3\left|\begin{array}{lll}
f_{1}^{0} & \frac{\partial^{2} f_{1}}{\partial \lambda^{2}} & \frac{\partial^{3} f_{1}}{\partial \lambda^{2} \partial \mu} \\
f_{2}^{0} & \frac{\partial^{2} f_{2}}{\partial \lambda^{2}} & \frac{\partial^{3} f_{2}}{\partial \lambda^{2} \partial \mu} \\
f_{3}^{0} & \frac{\partial^{2} f_{3}}{\partial \lambda^{2}} & \frac{\partial^{3} f_{3}}{\partial \lambda^{2} \partial \mu}
\end{array}\right|+\left|\begin{array}{lll}
f_{1}^{0} & \frac{\partial f_{1}}{\partial \lambda} & \frac{\partial^{4} f_{1}}{\partial \lambda^{3} \partial \mu} \\
f_{2}^{0} & \frac{\partial f_{2}}{\partial \lambda} & \frac{\partial^{4} f_{2}}{\partial \lambda^{3} \partial \mu} \\
f_{3}^{0} & \frac{\partial f_{3}}{\partial \lambda} & \frac{\partial^{4} f_{3}}{\partial \lambda^{3} \partial \mu}
\end{array}\right| .
\end{gathered}
$$


Von diesen Determinanten verschwinden hier die erste und die letzte für $\frac{\lambda}{\mu}=\frac{\lambda^{0}}{\mu^{0}}$ wegen der Gleichungen (4.). Die Summe der beiden anderen kann man zugleich in der Form darstellen:

$$
\begin{gathered}
\left\{\frac{\partial}{\partial \lambda}\left|\begin{array}{ccc}
f_{1} & \frac{\partial^{2} f_{1}}{\partial \lambda^{2}} & \frac{\partial^{2} f_{1}}{\partial \lambda \partial \mu} \\
f_{2} & \frac{\partial^{2} f_{2}}{\partial \lambda^{2}} & \frac{\partial^{2} f_{2}}{\partial \lambda \partial \mu} \\
f_{3} & \frac{\partial^{2} f_{3}}{\partial \lambda^{2}} & \frac{\partial^{2} f_{3}}{\partial \lambda \partial \mu}
\end{array}\right|-\left.\left|\begin{array}{lll}
\frac{\partial f_{1}}{\partial \lambda} & \frac{\partial^{2} f_{1}}{\partial \lambda^{2}} & \frac{\partial^{2} f_{1}}{\partial \lambda \partial \mu} \\
\frac{\partial f_{2}}{\partial \lambda} & \frac{\partial^{2} f_{2}}{\partial \lambda^{2}} & \frac{\partial^{2} f_{2}}{\partial \lambda \partial \mu} \\
\frac{\partial f_{3}}{\partial \lambda} & \frac{\partial^{2} f_{3}}{\partial \lambda^{2}} & \frac{\partial^{2} f_{3}}{\partial \lambda \partial \mu}
\end{array}\right|\right|_{\lambda=\lambda^{0}, \mu=\mu^{\prime \prime}}\right. \\
=\left(\frac{1}{n \cdot n-1} \frac{\partial \cdot \lambda^{2} \Delta}{\partial \lambda}\right)_{\lambda=\lambda^{0}, \mu=\mu^{0}},
\end{gathered}
$$

was nach dem Früheren für einen Rückkehrpunkt ebenfalls verschwindet. Das Verschwinden des ersten und zweiten Differentialquotienten der linken Seite von (16.) wird noch leichter ähnlich gezeigt; es ist also wirklich $\frac{\lambda^{0}}{\mu^{0}}$ hier eine vierfache Wurzel. Aber unter diesen ist schon eine der $\varkappa$ Wurzeln enthalten, die wegen der $\varkappa$ Rückkehrpunkte in Abzug zu bringen sind. Man sieht also, dass sich von einem Rückkehrpunkte noch $2 n-5-\varkappa$ Tangenten an die Curve ziehen lassen.

Es bleiben endlich die Doppeltangenten zu untersuchen. Sind die Parameter ihrer Berührungspunkte $\frac{\lambda}{\mu}, \frac{\lambda^{0}}{\mu^{0}}$, so finden nach (16.), (17.) die beiden Gleichungen statt:

$$
\left\{\begin{array}{c}
\frac{1}{\left(\lambda \mu^{0}-\lambda^{0} \mu\right)^{2}} \cdot\left|\begin{array}{lll}
f_{1}^{0} & \frac{\partial f_{1}}{\partial \lambda} & \frac{\partial f_{1}}{\partial \mu} \\
f_{2}^{0} & \frac{\partial f_{2}}{\partial \lambda} & \frac{\partial f_{2}}{\partial \mu} \\
f_{3}^{0} & \frac{\partial f_{3}}{\partial \lambda} & \frac{\partial f_{3}}{\partial \mu}
\end{array}\right|=-n \Psi\left(\lambda, \mu ; \lambda, \mu ; \lambda^{0}, \mu^{0}\right), \\
\frac{1}{\left(\lambda \mu^{0}-\lambda^{0} \mu\right)^{2}} \cdot\left|\begin{array}{lll}
f_{1} & \frac{\partial f_{1}^{0}}{\partial \lambda^{0}} & \frac{\partial f_{1}^{0}}{\partial \mu^{0}} \\
f_{2} & \frac{\partial f_{2}^{0}}{\partial \lambda^{0}} & \frac{\partial f_{2}^{0}}{\partial \mu^{0}} \\
f_{3} & \frac{\partial f_{3}^{0}}{\partial \lambda^{0}} & \frac{\partial f_{8}^{0}}{\partial \mu^{0}}
\end{array}\right|=-n \Psi\left(\lambda^{0}, \mu^{0} ; \lambda^{0}, \mu^{0} ; \lambda, \mu\right) .
\end{array}\right.
$$


54 Clebsch, über Curven, deren Coord. rationale Funct. eines Parameters sind.

Von diesen Gleichungen ist die erste vom $(n-2)^{\text {ten }}$, die zweite vom $2(n-2)^{\text {ten }}$ Grade für $\frac{\lambda^{0}}{\mu^{0}}$, und die erste vom $2(n-2)^{\text {ten }}$, die zweite vom $(n-2)^{\text {ten }}$ Grade für $\frac{\lambda}{\mu}$. Eliminirt man also eine dieser Grössen etwa $\frac{\lambda^{0}}{\mu^{0}}$, so erhält man eine Gleichung in $\frac{\lambda}{\mu}$ vom Grade $5(n-2)^{2}$. Aber diese Gleichung enthält eine Reihe von Factoren, welche der Frage fremd sind.

Erstlich ist das Eliminationsresultat, nach einer schon von Abel in Bezug auf solche Gleichungen gemachten Bemerkung, durch $\Psi(\lambda, \mu ; \lambda, \mu ; \lambda, \mu)$, d. h. durch $\Delta$ theilbar, so dass der Rest vom Grade $5(n-2)^{2}-3(n-2)$ bleibt. Ferner ist sie theilbar durch die Gleichung $\psi(\lambda, \mu)=0$, von welcher die Doppelpunkte abhängen, weil mit den Gleichungen (11.) auch die Gleichungen (18.) erfüllt sind. Der übrigbleibende Factor ist dann vom Grade $5(n-2)^{2}-3(n-3)-(n-1)(n-2)=4(n-2)(n-3)$.

Diese Zahl erhält aber eine weitere Reduction, wenn Rückkehrpunkte vorhanden sind. Die Gleichưgen (18.) werden nämlich offenbar noch erfüllt, wenn $\frac{\lambda^{0}}{\mu^{0}}$ und $\frac{\lambda}{\mu}$ zwei verschiedenen Rückkehrpunkten entsprechen, und wenn eine dieser Grössen einem Rückkehrpunkte entspricht, die andere dem Berührungspunkte einer von einem Rückkehrpunkte aus gezogenen Tangente. $\mathrm{Zu}$ einem Werthe $\frac{\lambda}{\mu}$, welcher einem Rückkehrpunkte entspricht, gehören also noch $\varkappa-1+2 n-5-\varkappa$ Werthe $\frac{\lambda^{0}}{\mu^{0}}$, welche der übrig gebliebenen Gleichung genügen; jeder solche Werth $\frac{\lambda}{\mu}$ ist also eine $(2 n-6)$ fache Wurzel der Gleichung. Und jeder Werth $\frac{\lambda}{\mu}$, der einer von einem Rückkehrpunkte gezogenen Tangente entspricht, genügt jener Gleichung ebenfalls. Sie enthält also noch zwei fremde Factoren, beziehungsweise von den Graden $\varkappa(2 n-6)$ und $(2 n-5-\varkappa) \varkappa$, so dass nach Ausscheidung derselben eine Gleichung vom Grade

$$
4(n-3)(n-2-x)+x(x-1)
$$

zurückbleibt. Die Anzahl aller Doppeltangenten ist also

$$
2(n-3)(n-2-x)+\frac{x \cdot x-1}{2}
$$

und da je zwei zusammengehörige Wurzeln der soeben aufgestellten Gleichung aus (18.) rational durch einander ausgedrückt werden können, so findet man diese Doppeltangenten durch eine Gleichung des Grades $2(n-3)(n-2-x)+\frac{x \cdot x-1}{2}$, und die Berührungspunkte jeder einzelnen durch eine quadratische Gleichung. 
Die angegebenen Zahlen stimmen mit denjenigen überein, welche aus den Plikckerschen Gleichungen folgen. Aber es ist von Interesse auf dem vorliegenden Wege gesehen zu haben, wie man die zur Auffindung dieser Singularitäten nöthigen Gleichungen wirklich zu bilden, und durch Gleichungen von möglichst niedrigem Grade aufzulösen hat.

Dass die Curve (1.) wirklich die allgemeinste ist, welche $\frac{n-1 . n-2}{2}$ Doppelpunkte besitzt, kann man sich auch folgendermassen deutlich machen. Die Ausdrücke (1.) enthalten $3 n+3$ willkürliche Constanten. Durch eine lineare Transformation von der Form

$$
\frac{\lambda}{\mu}=\frac{p \lambda^{\prime}+q \mu^{\prime}}{r \lambda^{\prime}+s \mu^{\prime}}
$$

kann man diese auf $3 n-1$ reduciren. Aber es ist

$$
3 n-1=\frac{n . n+3}{2}-\frac{n-1 . n-2}{2},
$$

d. h. gleich der Anzahl Bestimmungsstücke, welche eine allgemeine Curve $\boldsymbol{n}^{\text {ter }}$ Ordnung fordert, weniger der Anzahl von Bedingungen, welcher die geforderte Anzahl von Doppelpunkten mit sich führt. Die Anzahl von willkürlichen Constanten ist also ebenso gross, wie die Zahl der Bedingungen, welche die allgemeinste Curve der gegebenen Art noch zu erfüllen im Stande ist.

\section{\$. 2 .}

Das Abelsche Theorem und das Problem der Abelschen Functionen für den Fall, in welchem die Abelschen Integrale durch Logarithmen und algebraische Functionen ausdrückbar sind.

Im Folgenden setze ich $\mu=1$, da die Differentiation nach dieser Grösse die Beibehaltung des Zeichens nicht mehr erheischt. Sodann bezeichne ich durch $a^{(1)}, b^{(1)} ; a^{(2)}, b^{(2)} ; \ldots a^{(v)}, b^{(v)}\left(\nu=\frac{n-1 . n-2}{2}\right)$ die den verschiedenen Doppelpunkten entsprechenden Werthepaare von $\lambda$. Die Durchschnitte der gegebenen Curve mit einer Curve $m^{\text {ter }}$ Ordnung

$$
\text { (19.) } \varphi\left(x_{1}, x_{2}, x_{3}\right)=0
$$

werden gefunden, indem man in dieser Gleichung für die $x$ ihre Werthe (1.) setzt. Man erhält dann eine Gleichung in $\lambda$ :

(20.) $\Omega(\lambda) \equiv c\left(\lambda-\lambda_{1}\right)\left(\lambda-\lambda_{2}\right) \ldots\left(\lambda-\lambda_{n m}\right) \equiv \varphi\left(x_{1}, x_{2}, x_{3}\right)=0$,

wo $\lambda_{1}, \lambda_{2}, \ldots \lambda_{n m}$ den $n m$ Durchschnittspunkten entsprechen. Setzen wir in 
(20.) $\lambda$ einmal $a^{(i)}$, das andere Mal $b^{(i)}$, so bleiben die $x$ bis auf einen gemeinschaftlichen Factor $c^{(i)}$, also auch $\varphi$ und $\Omega$ bis auf den Factor $c^{(i)^{m}}$ dadurch ungeändert, und man erhält $\Omega\left(a^{(i)}\right)=c^{(i)^{m}} \Omega\left(b^{(i)}\right)$, oder

$$
\text { (21.) } \quad \frac{a^{(i)}-\lambda_{1} \cdot a^{(i)}-\lambda_{2} \ldots a^{(i)}-\lambda_{n m}}{b^{(i)}-\lambda_{1} \cdot b^{(i)}-\lambda_{2} \ldots b^{(i)}-\lambda_{n m}}=c^{(i)^{m}} \text {. }
$$

Diese Gleichung, welche $\nu$ verschiedene vertritt, ist für die Theorie der vorliegenden Curven von der höchsten Wichtigkeit. Sie vertritt für diese Curven die Stelle derjenigen, welche ich im allgemeinen Falle aus dem Abelschen Theoreme abgeleitet habe (dieses Journal Band 63, pag. 189). Die $v$ Gleichungen (21.) versehen die Stelle der $\nu$ Relationen, welche erfüllt sein müssen damit die $n m$ Schnittpunkte wirklich auf einer Curve $m^{\text {ter }}$ Ordnung liegen; und man hat daher den Satz:

Wenn nm Punkte der Curve (1.) auf einer Curve $m^{\text {ter }}$ Ordnung liegen sollen, so ist es nöthig und hinreichend, dass ihre Parameter den Gleichungen (21.) genügen.

Die Gleichung (21.) erhält eine leichte Modification, wenn einer der Doppelpunkte in einen Rückkehrpunkt übergeht. Man hat dann $a^{(i)}=\alpha^{(i)}$, $b^{(i)}=\alpha^{(i)}+\varepsilon, c^{(i)}=1-\varkappa^{(i)} \varepsilon$, wo $\varepsilon$ unendlich klein ist, und also

$$
\frac{a^{(i)}-\lambda}{b^{(i)}-\lambda}=1-\frac{\varepsilon}{a^{(i)}-\lambda} \cdots
$$

Die Gleichung (21.) geht daher über in folgende:

$$
\frac{1}{\alpha^{(i)}-\lambda_{1}}+\frac{1}{\alpha^{(i)}-\lambda_{2}}+\cdots+\frac{1}{\alpha^{(i)}-\lambda_{n m}}=m \varkappa^{(i)} \text {. }
$$

Die Integralsummen, welche, gleich Null gesetzt, die Stelle dieser Gleichungen vertreten (a. a. 0.p. 197) findet man, indem man (21.) (22.), ersteres logarithmisch, differentiirt, und dann von $\varphi^{(i)}$ bis $\lambda$, beziehungsweise $\sigma^{(i)}$ bis $\lambda$, integrirt. Man findet so statt (21.) (22.) die folgenden Gleichungen:

$$
\left\{\begin{array}{l}
\int_{e^{(i)}}^{\lambda_{1}} \frac{\left(a^{(i)}-b^{(i)}\right) d \lambda}{a^{(i)}-\lambda \cdot b^{(i)}-\lambda}+\int_{\rho^{(i)}}^{\lambda_{2}} \frac{\left(a^{(i)}-b^{(i)}\right) d \lambda}{a^{(i)}-\lambda \cdot b^{(i)}-\lambda}+\cdots+\int_{\rho^{(i)}}^{\lambda_{n m}} \frac{\left(a^{(i)}-b^{(i)}\right) d \lambda}{a^{(i)}-\lambda \cdot b^{(i)}-\lambda}=0 \\
\int_{\sigma^{(i)}}^{\lambda_{1}} \frac{d \lambda}{\left(a^{(i)}-\lambda\right)^{2}}+\int_{\sigma^{(i)}}^{\lambda_{2}} \frac{d \lambda}{\left(a^{(i)}-\lambda\right)^{2}}+\cdots+\int_{\sigma^{(i)}}^{\lambda_{n m}} \frac{d \lambda}{\left(a^{(i)}-\lambda\right)^{2}}=0,
\end{array}\right.
$$

und die unteren Grenzen $\varrho$, $\sigma$ bestimmen sich aus der Vergleichung mit (21.), (22.) durch die Gleichungen:

$$
\text { (24.) } \quad \frac{a^{(i)}-\varrho^{(i)}}{b^{(i)}-\varrho^{(i)}}=\left(c^{(i)}\right)^{\frac{1}{n}}, \quad \frac{1}{a^{(i)}-\sigma^{(i)}}=\frac{x^{(i)}}{n} .
$$


Durch Veränderung des Integrationsweges kann man auf der rechten Seite der ersten dieser Gleichungen 0 in $2 m i \pi$ verwandeln, so dass diese Integralsummen die Periode 2iл zulassen. Die Integrale der zweiten Gleichung aber haben immer die Periode Null, da sie nur paarweise zusammenfallende Unstetigkeitspunkte besitzen. Dies kommt damit überein, dass man rechts in (22.) $\left(c^{(i)}\right)^{m} \cdot e^{2 h i \pi}$ für $\left(c^{(i)}\right)^{m}$ setzen kann, während $m \varkappa^{(i)}$ auf der rechten Seite von (23.) sich durch nichts Anderes ersetzen lässt. Man kann also folgenden Satz aussprechen:

Für eine Curve $n^{\text {ter }}$ Ordnung mit $\frac{n-1 . n-2}{2}$ Doppelpunkten, respective Rückkehrpunkten, ist, wenn in der Darstellung (1.) $a^{(i)}, b^{(i)}$ die Parameter eines Doppelpunktes sind, immer die Summe gleichartiger Integrale

$$
\int_{e}^{\lambda} \frac{d \lambda\left(a^{(i)}-b^{(i)}\right)}{a^{(i)}-\lambda \cdot b^{(i)}-\lambda}
$$

gleich 2his, hingegen, wenn $\alpha^{(i)}$ der Parameter eines Rückkehrpunktes ist, die Summe der Integrale

$$
\int_{\sigma}^{\lambda} \frac{d \lambda}{\left(\alpha^{(i)}-\lambda\right)^{2}}
$$

gleich Null; wenn die Summen auf alle Parameter erstreckt werden, die den Schnittpunkten der Curve mit einer algebraischen Curve entsprechen.

Hieraus geht sofort hervor, dass alle Aufgaben, welche ich a. a. 0. mit Hülfe der Theilung der Abelschen Transcendenten gelöst habe, für die vorliegenden Curven auf Kreistheilung zurückkommen, und auf die Auflösung einer Gleichung $\boldsymbol{v}^{\text {ten }}$ Grades.

In der That lässt sich dasselbe aus den Gleichungen (22.), (23.) direct nachweisen. In allen jenen Aufgaben sind nämlich $n m-\mu \nu$ Schnittpunkte gegeben, während von den übrigen $\nu$ mal $\mu$ zusammenfallen sollen. Bezeichnen wir die den ersten entsprechenden (gegebenen) Parameter durch $l_{1}, l_{2}, \ldots l_{n m-\mu \nu}$, die der gesuchten Punkte durch $\lambda_{1}, \lambda_{2} \ldots \lambda_{\nu}$, so gehen die Gleichungen (22.) über in:

$$
\left\{\begin{array}{l}
\frac{a^{(i)}-\lambda_{1} \cdot a^{(i)}-\lambda_{2} \ldots a^{(i)}-\lambda_{\nu}}{b^{(i)}-\lambda_{1} \cdot b^{(i)}-\lambda_{2} \ldots b^{(i)}-\lambda_{\nu}}=e^{\frac{2 h^{(i)} i_{i n}}{\mu}} \sqrt[\mu]{c^{(i)^{m}} \cdot \frac{b^{(i)}-l_{1} \cdot b^{(i)}-l_{2} \ldots b^{(i)}-l_{n m-\mu \nu}}{a^{(i)}-l_{1} \cdot a^{(i)}-l_{2} \ldots a^{(i)}-l_{m n-\mu \nu}},} \\
\frac{1}{\alpha^{(i)}-\lambda_{1}}+\frac{1}{\alpha^{(i)}-\lambda_{2}}+\cdots+\frac{1}{\alpha^{(i)}-\lambda_{\nu}}=\frac{1}{\mu}\left\{m \varkappa^{(i)}-\frac{1}{\alpha^{(i)}-l_{1}}-\frac{1}{\alpha^{(i)}-l_{2}}-\cdots-\frac{1}{\alpha^{(i)}-l_{n m-\mu \nu}}\right\} .
\end{array}\right.
$$

Diese Gleichungen, deren man im Ganzen $\nu$ hat, genügen völlig, um die symmetrischen Functionen der $\lambda$ durch bekannte Grössen auszudrücken, und eine Gleichung $\boldsymbol{\nu}^{\text {ten }}$ Grades für dieselben anzusetzen. 
Man bemerkt, dass der Einfluss eines Rückkehrspunktes darin besteht, eine der $\nu$ Perioden verschwinden zu machen. Wenn, wie dies bei den Curven vierter Ordnung noch geschehen kann, sämmtliche Doppelpunkte in Rückkehrpunkte übergehen, so hört die Benutzung der Kreistheilung überhaupt auf.

Dass hierher gehörige Umkehrungsproblem ist in den Gleichungen enthalten:

$$
\text { (25.) } \sum_{h=1}^{h=\frac{n-1 . n-2}{2}} \int_{\rho}^{\lambda} \frac{\left(a^{(i)}-b^{(i)}\right) d \lambda}{a^{(i)}-\lambda \cdot b^{(i)}-\lambda}=u^{(i)} \quad\left(i=1,2 \cdots \frac{n-1 . n-2}{2}\right),
$$

deren einige auch ersetzt sein können durch Gleichungen von der Form

$$
\text { (26.) } \sum_{l=1}^{h=\frac{n-1, n-2}{2}} \int_{\sigma}^{\lambda_{h}} \frac{d \lambda}{\left(\alpha^{(i)}-\lambda\right)^{2}}=v^{(i)} \text {, }
$$

welche aus jener hervorgeht, wenn $a^{(i)}=\alpha^{(i)}, b^{(i)}=\alpha^{(i)}+\varepsilon, v^{(i)}=-\frac{u_{(i)}}{\varepsilon}$ gesetzt wird. Die Auflösung des Umkehrungsproblems ist sodann durch die Gleichungen gegegeben $\left(v=\frac{n-1 . n-2}{2}\right)$ :

$$
\text { (27.) } \quad \frac{a^{(i)}-\lambda_{1} \cdot a^{(i)}-\lambda_{2} \ldots a^{(i)}-\lambda_{\nu}}{b^{(i)}-\lambda_{1} \cdot b^{(i)}-\lambda_{2} \ldots b^{(i)}-\lambda_{\nu}}=e^{u^{(i)}}\left(\frac{a^{(i)}-\varrho}{b^{(i)}-\varrho}\right)^{\nu}=e^{u^{(i)}}\left(c^{(i)}\right)^{\frac{\nu}{n}}
$$

nur wenn der Doppelpunkt $a, b$ in einen Rückkehrpunkt $\alpha$ übergeht, ist diese Gleichung zu ersetzen durch

$$
\left(27^{a} .\right) \quad \frac{1}{\alpha^{(i)}-\lambda_{1}}+\frac{1}{\alpha^{(i)}-\lambda_{2}}+\cdots+\frac{1}{\alpha^{(i)}-\lambda_{\nu}}=v^{(i)}+\frac{\nu}{\alpha^{(i)}-\sigma^{(i)}}=v^{(i)}+\frac{\nu x^{(i)}}{n} .
$$

Die Grössen $c$, also auch die $\varrho$, drückt man leicht durch die $a, b$ aus. Man braucht nämlich nur zu bemerken, dass, wenn man einen Doppelpunkt absondert, die übrigen den vollständigen Durchschnitt der Curve $n^{\text {ter }}$ Ordnung mit der Curve $n-3^{\text {ter }}$ Ordnung bilden, welche sich durch jene $\frac{n . n-3}{2}$ Doppelpunkte legen lässt. Setzt man also in (21.) $m=n-3$, so kann man zugleich den $n . n-3$ Grössen $\lambda$ die $n . n-3$ Werthe $a, b$ beilegen, welche den Index $i$ nicht haben; es besteht also, wenn man der Kürze wegen

setzt, die Gleichung:

$$
\left\{\begin{array}{l}
z-a^{(1)} \cdot z-a^{(2)} \ldots z-a^{(\nu)}=\varphi(z) \\
z-b^{(1)} \cdot z-b^{(2)} \ldots z-b^{(\nu)}=\psi(z)
\end{array}\right.
$$

(29.) $\quad\left(c^{(i)}\right)^{n-3}=-\frac{\varphi^{\prime}\left(a^{(i)}\right) \cdot \psi\left(a^{(i)}\right)}{\psi^{\prime}\left(b^{(i)}\right) \cdot \varphi\left(b^{(i)}\right)}$. 
Untersuchen wir nun, unter welchen Umständen ein System von Grössen $u^{(i)}$ Summen von nur $\nu-1$ Integralen (25.) gleich werden kann. In diesem Falle ist an Stelle von (27.) die Gleichung zu setzen:

$$
\left\{\begin{array}{c}
\frac{a^{(i)}-\lambda_{1} \cdot a^{(i)}-\lambda_{2} \ldots a^{(i)}-\lambda_{\nu-1}}{b^{(i)}-\lambda_{1} \cdot b^{(i)}-\lambda_{2} \ldots b^{(i)}-\lambda_{\nu-1}}=e^{u^{(i)}} \cdot\left(c^{(i)}\right)^{\frac{\nu-1}{n}}=e^{u^{(i)}} \cdot\left(c^{(i)}\right)^{\frac{n-3}{2}} \\
=e^{u^{(i)}} \cdot \sqrt{-\frac{\varphi^{\prime}\left(a^{(i)}\right) \cdot \psi\left(a^{(i)}\right)}{\psi^{\prime}\left(b^{(i)}\right) \cdot \varphi\left(b^{(i)}\right)}}=\zeta^{(i)} .
\end{array}\right.
$$

Dieses sind $\nu$ Gleichungen, aus denen man die $\nu-1$ symmetrischen Functionen der $\lambda$ eliminiren kann, und zwar wird das Resultat der Elimination die Gleichung:

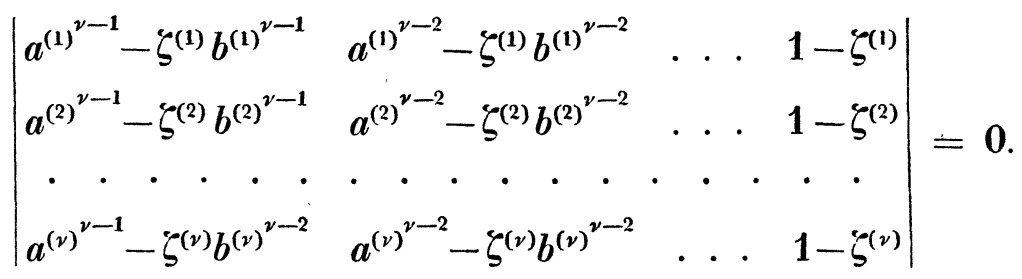

Ordnet man diese Gleichung nach den $\zeta$, und ersetzt dieselben durch die in (30.) gegebenen Werthe, so ergiebt sich eine Gleichung in den $e^{n}$, welche jede dieser Grössen nur auf lineare Weise enthält. Bezeichnet man nun die Grössen $e^{u}$ in irgend welcher Folge durch die Indices $\alpha_{1}, \alpha_{2}, \ldots \alpha_{i}, \beta_{i+1}, \beta_{i+2}, \ldots \beta_{v}$, so ist leicht zu zeigen, dass die in der Entwicklung von (31.) auftretenden Glieder

$$
e^{u^{\left(\alpha_{1}\right)}+u^{\left(\alpha_{2}\right)}+\cdots+u^{\left(\alpha_{i}\right)}}, \quad e^{u^{\left(\beta_{i+1}\right)}+u^{\left(\beta_{i+2}\right)}+\cdots+u^{\left(\beta_{\nu}\right)}}
$$

gleiche aber entgegengesetzte Coefficienten besitzen, und dass man also der Gleichung (31.) die Form geben kann

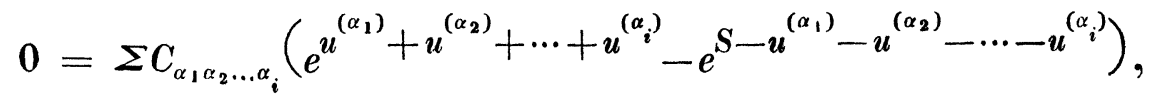

wo $S$ die Summe aller $u$ bezeichnet.

In der That: der Coefficient von $e^{u^{\left(\alpha_{1}\right)}+u^{\left(\alpha_{2}\right)}+\cdots+u^{\left(\alpha_{i}\right)}}$ in (31.) ist $(-1)^{i}$ multiplicirt erstlich in den Ausdruck, welcher entsteht, wenn man in der Determinante

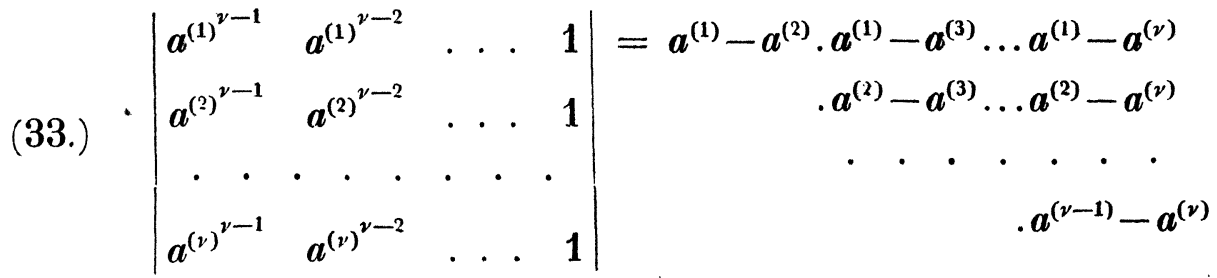


die mit einem Index $\alpha$ behafteten $a$ durch die betreffenden $b$ ersetzt; multiplicirt zweitens in das Product:

Der Coefficient von

$$
e^{S-u^{\left(\alpha_{1}\right)}-u^{\left(\alpha_{2}\right)} \cdots}=e^{u^{\left(\beta_{i+1}\right)}+u^{\left(\beta_{i+2}\right)}+\cdots+u^{(\beta)}}
$$

unterscheidet sich von diesem dadurch, dass $(-1)^{\nu-i}$ an Stelle von $(-1)^{i}$ tritt, dass in der Determinante die $a$ mit einem Jndex $\beta$ durch die entsprechenden $b$ ersetzt werden, und endlich, dass in dem Product der $c$ überall die Indices $\beta$ statt der Indices $\alpha$ auftreten. Statt dieses Productes aber denke ich mir das Product sämmtlicher $c^{\frac{n-3}{2}}$ gesetzt, dividirt durch den Ausdruck (33.).

Der Quotient der so gebildeten Coefficienten muss gleich 1 sein. Bezeichnen wir durch $\Pi$ Differenzenproducte, so nimmt nach dem Vorigen dieser Quotient folgenden Werth an:

$$
\begin{aligned}
& \frac{\Pi\left(b^{\left(\alpha_{i}\right)}-b^{\left(\alpha_{k}\right)}\right) \cdot \Pi\left(b^{\left(\alpha_{i}\right)}-a^{\left(\beta_{k}\right)}\right) \cdot \Pi\left(a^{\left(\beta_{i}\right)}-a^{\left(\beta_{k}\right)}\right)}{\Pi\left(a^{\left(\alpha_{i}\right)}-a^{\left(\alpha_{k}\right)}\right) \cdot \Pi\left(a^{\left(\alpha_{i}\right)}-b^{\left(\beta_{k}\right)}\right) \cdot \Pi\left(b^{\left(\beta_{i}\right)}-b^{\left(\beta_{k}\right)}\right) \cdot \frac{1}{\left(c^{(1)} \cdot c^{(2)} \ldots c^{(\nu)}\right)^{\frac{n-3}{2}}}} \\
& \cdot \frac{\Pi\left(a^{\left(\alpha_{i}\right)}-a^{\left(\alpha_{k}\right)}\right)^{2} \cdot \Pi\left(a^{\left(\beta_{i}\right)}-a^{\left(\alpha_{k}\right)}\right) \cdot \Pi\left(b^{\left(\alpha_{i}\right)}-a^{\left(\alpha_{k}\right)}\right) \cdot \Pi\left(b^{\left(\beta_{i}\right)}-a^{\left(\alpha_{k}\right)}\right)}{\Pi\left(b^{\left(\alpha_{i}\right)}-b^{\left(\alpha_{k}\right)}\right)^{2} \cdot \Pi\left(b^{\left(\beta_{i}\right)}-b^{\left(\alpha_{k}\right)}\right) \cdot \Pi\left(a^{\left(\alpha_{i}\right)}-b^{\left(\alpha_{k}\right)}\right) \cdot \Pi\left(a^{\left(\beta_{i}\right)}-b^{\left(\alpha_{k}\right)}\right)} .
\end{aligned}
$$

Aber dies geht sofort, wenn $A$ das Differenzenproduct sämmtlicher $a, B$ das sämmtlicher $b$ ist, über in

$$
\frac{A}{B} \cdot \frac{1}{\left(c^{(1)} \cdot c^{(2)} \ldots c^{(v)}\right)^{\frac{n-3}{2}}} .
$$

Dies ist wirklich 1, da, wie leicht zu sehen, das Product sämmlicher $c^{n-3}$ gleich $\frac{A^{2}}{B^{2}}$ ist, und also das Product aller $c^{\frac{n-3}{2}}$ gleich $\frac{A}{B}$, wenn nur, was immer geschehen kann, eine passende Combination der Vorzeichen der Quadratwurzeln vorausgesetzt wird.

Wir haben so den Satz:

Soll ein System von $\nu$ Grössen $u^{(i)}$ den Summen von je $(\nu-1)$ Integralen gleich werden, so ist es nöthig und hinreichend, dass zwischen den $u$ die Gleichung (31.) oder (32.) bestehe.

Sind insbesondere sämmtliche Grössen $u$ ganze Vielfache von $i \pi$, also alle Grössen $e^{u}$ gleich \pm 1 , so reducirt sich die Gleichung (32.) auf

(35.) $e^{s}=1$, 
d. h. die Summe sämmtlicher $u$ muss dann einem geraden Vielfachen von iл gleich sein.

Es bleiben die Modificationen zu untersuchen, welche diese Betrachtungen erfahren, wenn einige Doppelpunkte in Rückkehrpunkte übergehen. Was zunächst die Bestimmung der den wirklichen Doppelpunkten entsprechenden $c$ betrifft, so hebt sich in der Formel (29.) alles von den Rückkehrpunkten Herrührende (wo $a=b=\alpha$ ) einfach auf, und es genügt also dann, die Functionen $\varphi, \psi$ auf diejenigen Factoren zu beschränken, die den wirklichen Rückkehrpunkten entsprechen. Die $\varkappa$ bestimmt man aus (22.), wie oben die $c$ aus (21.), und findet also, wenn noch

gesetzt wird :

$$
\text { (36.) } \quad \chi(z)=z-\alpha^{(1)} . z-\alpha^{(2)} \ldots
$$

$$
\text { (37.) } \quad \varkappa^{(i)}=\frac{1}{n-3}\left\{\frac{\chi^{\prime \prime}\left(\alpha^{(i)}\right)}{2 \chi^{\prime}\left(\alpha^{(i)}\right)}+\frac{\varphi^{\prime}\left(\alpha^{(i)}\right)}{\varphi\left(\alpha^{(i)}\right)}+\frac{\psi^{\prime}\left(\alpha^{(i)}\right)}{\psi\left(\alpha^{(i)}\right)}\right\} .
$$

Ferner tritt, wenn man die Substitutionen $u^{(i)}=-\varepsilon v^{(i)}, c^{(i)}=1-\varepsilon \psi^{(i)}, a^{(i)}=\alpha^{(i)}$, $b^{(i)}=\alpha^{(i)}+\varepsilon$ macht, und $\varepsilon$ gegen Null convergiren lässt, an Stelle der betreffenden Reihe

$$
a^{\nu-1}-\zeta b^{\nu-1}, \quad a^{\nu-2}-\zeta b^{\nu-2}, \ldots a-\zeta b, 1-\zeta
$$

in (31.) immer eine Reihe von der Form:

wo

$$
\alpha^{\nu-2}-\vartheta \alpha^{\nu-1}, 2 \alpha^{\nu-3}-\vartheta \alpha^{\nu-2}, \ldots(\nu-1)-\vartheta \alpha,-\vartheta
$$

$$
\text { (38.) } \boldsymbol{\vartheta}^{(i)}=v^{(i)}+\frac{n-3}{2} \varkappa^{(i)} .
$$

Sind insbesondere die $v$ sämmtlich gleich Null, und die $u$ ganze Vielfache von $i \pi$, so zeigt sich aus (35.), dass alsdann die Summe aller den Doppelpunkten entsprechenden $u$ ein gerades Vielfaches von iл sein muss.

\$. 3 .

Geometrische Resultate.

Nach diesen allgemeinen Vorbereitungen ist es leicht, die Modificationen anzugeben, welche die Lösungen der a. a. 0. behandelten Probleme in dem vorliegenden Falle erfahren.

Die Lösung dieser Probleme stützt sich immer auf die Gleichungen (21.), (22.):

(39.) $\left\{\begin{array}{l}\frac{a^{(i)}-\lambda_{1} \cdot b^{(i)}-\lambda_{2} \ldots b^{(i)}-\lambda_{n m}}{b^{(i)}}=c^{(i)^{m}} \\ \frac{1}{\alpha^{(i)}-\lambda_{1}}+\frac{1}{\alpha^{(i)}-\lambda_{2}}+\cdots+\frac{1}{\alpha^{(i)}-\lambda_{n m}}=m \varkappa^{(i)} .\end{array}\right.$ 
Die erste besteht für $\frac{n-1 . n-2}{2}-\varkappa$ Werthe von $\lambda$, die zweite, den Rückkehrpunkten entsprechend, für $*$ Werthe.

I. Für $m \bar{\equiv}-2$ ist folgende Aufgabe immer lösbar:

Es seien $m n-v r\left(v=\frac{n-1 . n-2}{2}\right)$ Punkte auf der Curve $n^{\text {ter }}$ Ordnung ge-

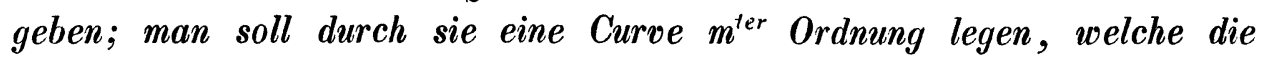
Curve $n^{\text {ter }}$ Ordnung in $\nu$ Punkten $r$-punktig beriuhrt.

Hier sind $m n-\nu r$ Grössen $\lambda$ gegeben; von den übrigen $v r$ sollen je $r$ zusammenfallen. Aus (39.) sind also, wenn $\lambda_{1}, \lambda_{2}, \ldots \lambda_{v}$ die den Berührungspunkten entsprechenden Parameter sind, die Ausdrücke

$$
\left(\frac{a^{(i)}-\lambda_{1} \cdot a^{(i)}-\lambda_{2} \ldots a^{(i)}-\lambda_{\nu}}{b^{(i)}-\lambda_{1} \cdot b^{(i)}-\lambda_{2} \ldots b^{(i)}-\lambda_{\nu}}\right)^{r}, \quad r\left(\frac{1}{\alpha^{(i)}-\lambda_{1}}+\frac{1}{\alpha^{(i)}-\lambda_{2}}+\cdots+\frac{1}{\alpha^{(i)}-\lambda_{\nu}}\right)
$$

gegeben, und man findet die $\lambda$ aus einer Gleichung $\nu^{\text {len }}$ Grades. Da man den ersten der obigen Ausdrücke durch Ausziehen einer $r^{\text {ten }}$ Wurzel bestimmt, und da dieselbe Operation $\nu-\varkappa$ mal vorkommt, so ist die Gesammtzahl aller - Lösungen des. Problems gleich $r^{\nu-x}$.

Bezeichnen wir die gegebenen Parameter durch $l_{1}, l_{2}, \ldots l_{n m-\nu r}$. Die Gleichungen, aus denen die $\lambda$ gefunden werden, sind dann:

$$
\begin{gathered}
\frac{a^{(i)}-\lambda_{1} \cdot a^{(i)}-\lambda_{2} \ldots a^{(i)}-\lambda_{v}}{b^{(i)}-\lambda_{1} \cdot b^{(i)}-\lambda_{2} \ldots b^{(i)}-\lambda_{\nu}} \sqrt{\frac{a^{(i)}-l_{1} \cdot a^{(i)}-l_{2} \cdots}{b^{(i)}-l_{1} \cdot b^{(i)}-l_{2} \cdots}}=e^{\frac{2 h^{(i)}{ }_{i \pi}}{r} c^{(i)}}, \\
\frac{1}{\alpha^{(i)}-\lambda_{1}}+\frac{1}{\alpha^{(i)}-\lambda_{2}}+\cdots+\frac{1}{\alpha^{(i)}-\lambda_{\nu}}+\frac{1}{r}\left(\frac{1}{a^{(i)}-l_{1}}+\frac{1}{u^{(i)}-l_{2}} \cdots\right)=\frac{m}{r} \varkappa^{(i)} .
\end{gathered}
$$

Denken wir uns diese Gleichungen für $r$ verschiedene Systeme von Lösungen hingeschrieben, und sodann die aus der ersten Gleichung hervorgehenden mit einander multiplicirt, die aus der zweiten hervorgehenden addirt. Ist dann nur die Summe der den verschiedenen Systemen entsprechenden $h$ durch $r$ theilbar (was immer durch passende Wahl der $h$ des letzten Systems erreicht wird), so sagen die resultirenden Gleichungen aus, dass die $r$ Systeme von Berührungspunkten mit den gegebenen Punkten auf einer Curve $m^{\text {ter }}$ Ordnung liegen. Man hat also den Satz:

Wenn man durch die gegebenen Punkte und durch die Berihrungspunkte von $r-1$ verschiedenen Berührungscurven eine Curve $m^{\text {ter }}$ Ordnung legt, so schneidet sie die gegebene Curve in solchen Punkten, in denen. wieder eine Berührungscurve beriihrt.

Dabei können die verschiedenen Curven, welche benutzt werden, ebensowohl verschieden als auch gleich sein; nur muss dann im letzteren Falle die Curve $m^{\text {ter }}$ Ordnung die gegebene Curve in entsprechender Weise berühren. 
II. Lässt man von den $m n-\nu r$ Punkten nur $m n-(\nu+\mu) r$ Punkte wirklich gegeben sein, und legt den übrigen ur nur die Bedingung auf, dass $\mu$ mal $r$ zusammenfallen sollen, so wird das Problem unbestimmt, und man erhält Systeme von Curven $m^{\text {ter }}$ Ordnung, welche durch $m n-(\nu+\mu) r$ gegebene Punkte der Curve $n^{\text {ter }}$ Ordnung gehen, und diese Curve in $(\nu+\mu)$ Punkten $r$-punktig berühren. Die verschiedenen Systeme unterscheiden sich dadurch, dass bei der Bestimmung der Ausdrücke

$$
\frac{a^{(i)}-\lambda_{1} \cdot a^{(i)}-\lambda_{2} \ldots a^{(i)}-\lambda_{\nu+\mu}}{b^{(i)}-\lambda_{1} \cdot b^{(i)}-\lambda_{2} \ldots b^{(i)}-\lambda_{\nu+\mu}}
$$

verschiedene $r^{\text {te }}$ Wurzeln der Einheit angewendet werden; die Anzahl aller Systeme ist also $r^{\nu-*}$. Ueber die Berührungspunkte besteht der oben bewiesene Satz fort.

III. Es kann endlich $m n-(\nu+\mu) r$ gleich Null werden, so dass die Systeme der Berührungscurve nur noch von der Natur der gegebenen Curve selbst abhängen. Ueber die Lage der Berührungspunkte gilt noch immer der vorige Satz; aber die Zahl der Systeme kann sich reduciren, wenn man alle diejenigen Systeme ausschliesst, deren Curven aus mehrfach gerechneten Curven niedrigerer Ordnung bestehen. Nach der Betrachtung des $\$$. 6 der angeführten Abhandlung, welche hier entsprechend dem unter I., II. eingeschlagenen Verfahren modificirt wird, bleibt, wenn $m=m^{\prime} s, r=r^{\prime} s$, und wenn $m^{\prime}, r^{\prime}$ relative Primzahlen sind, die Anzahl der eigentlichen Systeme $r^{\nu-x}-r^{\prime-x}$; und nur wenn $m, r$ relative Primzahlen sind, ist die Anzahl der Systeme wirklich gleich $r^{\nu-x}$.

IV. Im Vorigen wurde immer $m \equiv n-2$ vorausgesetzt. Für $m=n-3$ entsteht die Aufgabe:

Die Curve $n^{\text {ter }}$ Ordnung soll von einer Curve $(n-3)^{\text {ter }}$ Ordnung überall wo sie derselben begegnet, also in $\frac{n . n-3}{2}$ Punkten, zweipunktig beriihrt werden.

Die Gleichungen (39.) nehmen für diesen Fall die Form an:

$$
\begin{gathered}
\frac{a^{(i)}-\lambda_{1} \cdot a^{(i)}-\lambda_{2} \ldots a^{(i)}-\lambda_{\nu-1}}{b^{(i)}-\lambda_{1} \cdot b^{(i)}-\lambda_{2} \ldots b^{(i)}-\lambda_{\nu-1}}=c^{(i) \frac{n-3}{2}} \cdot e^{h^{(i)}{ }_{i n}}, \\
\frac{1}{\alpha^{(i)}-\lambda_{1}}+\frac{1}{\alpha^{(i)}-\lambda_{2}}+\cdots+\frac{1}{\alpha^{(i)}-\lambda_{\nu-1}}=\frac{n-3}{2} \cdot *^{(i)} .
\end{gathered}
$$

Die Gleichungen sind um eine zahlreicher, als die unbekannten $\lambda$. Aber man hat den am Ende des $\$ .2$ behandelten Fall vor sich, wo alle Grössen $e^{u^{(i)}}$ 
gleich \pm 1 , alle $v^{(i)}$ gleich Null waren. Es ist daher für das Zusammenbestehen dieser Gleichungen nur die Bedingung zu erfüllen, dass die Summe aller $h$ gerade sei. Hiedurch wird das letzte $h$ bestimmt, wenn die übrigen beliebig gewählt sind. Die Anzahl aller Berührungscurven ist also

$$
2^{y-x-1}=2^{\frac{n . n-3}{2}-x} .
$$

Nur dann giebt diese Zählung kein richtiges Resultat, wenn der Exponent negativ wird, d. h. wenn alle Doppelpunkte in Rückkehrpunkte übergehen. Dies kann nur bei den Curven dritter und vierter Ordnung stattfinden, da die Anzahl der Rückkehrpunkte (vgl. \$. 1) nicht grösser sein kann als $\frac{3(n-2)}{2}$. Da nun das vorliegende Problem bei den Curven dritter Ordnung überhaupt nicht eintritt, so bleibt nur der Fall der Curven vierter Ordnung mit drei Rückkehrpunkten übrig; und die obige Abzählung ist durch das bekannte Resultat (vgl. auch \$. 1) zu ergänzen, dass diese Curven eine Doppeltangente besitzen.

Ueber die Lage der Berührungspunkte und die Beziehungen dieser Curven, welche die gegebenen überall zweipunktig berühren, findet man leicht Sätze wie in $\$$. 8. der angeführten Abhandlung.

Ich begnüge mich zum Schluss einige dieser Resultate für Curven dritter und vierter Ordnung zu specialisiren.

Eine Curve dritter Ordnung mit einem Doppelpunkte besitzt ein System von Kegelschnitten, deren jeder die Curve in 3 Punkten berührt. Legt man durch drei Berührungspunkte einen Kegelschnitt, so trifft derselbe die Curve in den Berührungspunkten eines neuen Berührungskegelschnitts. Ebenso besitzt diese Curve zwei Systeme von Curven dritter Ordnung, welche die Curve ' in drei Punkten dreipunktig berühren. Sie besitzt drei Grade, welche sie dreipunktig berühren (Wendetangenten).

Geht der Doppelpunkt in einen Rückkehrpunkt über, so giebt es noch ein System von Berührungskegelschnitten, kein System von dreipunktig berührenden Curven dritter Ordnung und eine Wendetangente.

Eine Curve vierter Ordnung mit drei Doppelpunkten besitzt vier Doppeltangenten, deren Berührungspunkte auf einem Kegelschnitt liegen. Sie besitzt sieben Systeme von Kegelschnitten, welche die Curve in 4 Punkten berühren. Die Berührungspunkte je zweier Kegelschnitte desselben Systems liegen auf einem Kegelschnitt. Drei dieser Systeme enthalten je zwei Paare von Doppeltangenten und können aus denselben abgeleitet werden; die vier übrigen ent- 
halten keine Doppeltangenten. Die Curve besitzt sodann acht Systeme von Curven dritter Ordnung, welche die Curve in 6 Punkten berühren. Legt man durch die Berührungspunkte einer Curve eine Curve dritter Ordnung, so schneidet sie in den Berührungspunkten einer anderen desselben Systems. Vier Systeme haben die Eigenschaft, dass die Berührungspunkte mit dem Berührungspunkte einer Doppeltangente in einem Kegelschnitte liegen, die anderen haben diese Eigenschaft nicht. Es giebt 26 Systeme von Curven dritter Ordnung, welche in vier Punkten dreipunktig berühren u. s. w.

Geht ein Doppelpunkt in einen Rückkehrpunkt über, so hat man noch zwei Doppeltangenten; es giebt dann drei Systeme von Berührungskegelschnitten, deren eines das Paar von Doppeltangenten enthält. Die Curve hat vier Systeme von Curven dritter Ordnung, welche in 6 Punkten berühren; zwei Systeme haben die Eigenschaft, dass ihre Berührungspunkte mit den Berührungspunkten einer Doppeltangente in einem Kegelschnitt liegen. Es giebt 8 Systeme von Curven dritter Ordnung, die in vier Punkten dreipunktig berühren $u$. s. w.

Bei zwei Rückkehrpunkten hat man noch eine Doppeltangente und ein System von Berührungskegelschnitten, deren Berührungspunkte aber mit denen der Doppeltangente nicht in einem Kegelschnitt liegen. Es giebt zwei Systeme von Curven dritter Ordnung, die in 6 Punkten berühren; die Berührungspunkte eines Systems liegen mit denen der Doppeltangente in einem Kegelschnitt. Es giebt zwei Systeme von Curven dritter Ordnung, die in vier Punkten dreipunktig berühren, u. s. w.

Endlich bei drei Rückkehrpunkten giebt es zwar noch eine Doppeltangente, aber kein System von Berührungskegelschnitten mehr. Es giebt ein System von Curven dritter Ordnung, die in 6 Punkten berühren, und die 6 Berührungspunkte liegen mit denen der Doppeltangente in einem Kegelschnitt. Ein System von dreipunktig berührenden Curven dritter Ordnung existirt nicht melir.

Giessen, den 28. Mai 1864. 